\title{
AS COMPLEXAS IMBRICAÇÕES ENTRE PATENTES DE FÁRMACOS E DOENÇAS NEGLIGENCIADAS: LIMITES E POSSIBILIDADES NA PERSPECTVA DA SAÚDE GLOBAL
}

\author{
THE COMPLEX INTERACTIONS BETWEEN DRUG \\ PATENTS AND NEGLECTED DISEASES: LIMITS AND \\ POSSIBILITIES IN THE GLOBAL HEALTH PERSPECTIVE
}

\author{
Isabel Christine Silva De Gregori' ${ }^{1}$ Larissa Melez Ruviaro² \\ Nathália Facco Rocha ${ }^{3}$
}

DOI: https://doi.org/10.37767/2591-3476(2020)12

\begin{abstract}
RESUMEN:
Podría decirse que la ciencia y la tecnología han demostrado ser grandes aliados para mantener la salud, a través de la producción de medicamentos y vacunas para garantizar la ausencia de enfermedades. Por lo tanto, es necesario investigar las posibles implicaciones entre el actual sistema internacional de patentes y el crecimiento exponencial de las enfermedades olvidadas, en vista de las perspectivas de la salud mundial. Para cumplir el objetivo propuesto, se utilizaron la teoría de la teoría sistémica y el método de enfoque deductivo. Este artículo ha sido estructurado en dos capítulos. El primero abordará los instrumentos contextuales de la salud global y la imbricación de enfermedades olvidadas. El segundo tratará con el sistema de patentes como un potencial para las enfermedades de negligencia. Sin embargo, se concluye que muchas enfermedades están más allá del interés de las industrias, ya que no representan una perspectiva lucrativa.
\end{abstract}

\begin{abstract}
Arguably, science and technology have proven to be great allies of maintaining health, through the production of medicines and vaccines to ensure the absence of disease. Therefore, it is necessary to investigate the possible implications between the current international patent system and the exponential growth of neglected diseases, in view of the perspectives of global health. In order to fulfill the proposed objective, the Systemic Theory Theory and the deductive approach method were used. This article has been structured into two chapters. The first will address the contextual instruments of global health and the imbrication of neglected diseases.
\end{abstract}

\footnotetext{
1 Doutora em Desenvolvimento Regional pela Universidade de Santa Cruz do Sul. Mestre em Integração Latino-Americana pela Universidade Federal de Santa Maria. Professora na Universidade Federal de Santa Maria. Coordenadora do Grupo de Pesquisa em Propriedade Intelectual na Contemporaneidade. Endereço eletrônico: isabelcsdg@gmail.com. ORCID iD: https://orcid.org/0000-0002-3251-946X.

2 Advogada. Mestranda em Direito pela Universidade Federal de Santa Maria/UFSM. Integrante do Grupo de Pesquisa em Propriedade Intelectual na Contemporaneidade. Graduada em Direito pela FADISMA. Endereço eletrônico: larissa_ruviaro@hotmail.com. ORCID iD: https://orcid.org/0000-00016201-8183.

3 Mestre em Direito pela Universidade Federal de Santa Maria/UFSM. Especialista em Direito do Trabalho, Processo do Trabalho e Direito Previdenciário pela Faculdade Estácio de Sá. Integrante do Grupo de Pesquisa em Propriedade Intelectual na Contemporaneidade. Graduada em Direito pela FADISMA. Endereço Eletrônico: nathaliafacco@hotmail.com. ORCID iD: https://orcid.org/0000-0003-4465-5802.
} 
The second will deal with the patent system as a potential for neglect diseases. However, it is concluded that many diseases are beyond the interest of industries as they do not represent a lucrative prospect.

PALABRAS CLAVE: Enfermedades desatendidas; Industrias farmacéuticas; Patentes; Propiedad intelectual; Salud global.

PALAVRAS-CHAVE: Doenças negligenciadas; Indústrias Farmacêuticas; Patentes; Propriedade Intelectual; Saúde Global.

KEY WORDS: Neglected diseases; Pharmaceutical industries; Patents; Intellectual property; Global health

\section{Introdução}

O Sistema Internacional de Propriedade Intelectual passou a ganhar força a partir da Segunda Guerra Mundial, momento em que se delineava um sistema econômico global responsável pela intensificação do processo de internacionalização da economia, marcado pelo fortalecimento das multinacionais e mudança na estrutura de instituições econômicas capitalistas. Os benefícios obtidos através da evolução científica permitiram uma alavanca na produção da indústria farmacêutica e, aliado a isto, a possibilidade de gozar de um mercado dotado de privilégios, de uma legislação que além de garantir o monopólio na utilização comercial de seus produtos também assegura e a exclusividade por um grande lapso de tempo.

Este "modelo" acabou imprimindo uma lógica de mercado que não contempla a possibilidade de acesso a determinados medicamentos por parte da população, afastando, portanto, a perspectiva de concretização do direito humano a saúde. Este fato se atribuí ao lastro de sustentação do sistema de patentes, pautado em sua racionalidade econômica, na qual a proteção conferida aos criadores, inventores, estimularia o processo que envolve o ciclo de inovação, secundarizando valores fundamentais como o direito à saúde e aos medicamentos.

A falta de acesso a medicamento se torna um desafio à Saúde Global diante do Acordo TRIPS que estabelece padrões mínimos de proteção sobre os direitos de propriedade intelectual e garante a concessão de patente para todos os setores tecnológicos, inclusive para o campo farmacêutico. Em consequência disso, uma fatia importante da população mundial ficam a margem e desprovidas da atenção da indústria farmacêutica. Para tanto, cumpre investigar as possíveis imbricações entre o sistema internacional de patentes e o crescimento exponencial das doenças negligenciadas, diante das perspectivas da saúde global.

Para cumprir o objetivo proposto utilizou-se a Teoria de Base Sistêmica, considerando a abordagem transdisciplinar, visto que a temática compreende múltiplos ramos de conhecimento, fazendo parte de uma unicidades contextual. Como método de abordagem adotou-se o dedutivo, pois será realizada uma análise descendente, posto que, inicialmente, analisar-se-á as doenças negligenciadas e o acesso a medicamentos em um contexto de saúde global para, posteriormente, direcionar-se ao sistema de 
patentes como um potencializador das doenças negligencias, sob o viés da manutenção do poder e do acúmulo de capital.

Partindo da metodologia adotada, estruturou-se o presente artigo em dois capítulos. O primeiro capítulo abordará os instrumentos contextuais de saúde global e a imbricação das doenças negligencias face ao (des)cumprimento da garantia de acesso à medicamentos. O segundo capítulo versará sobre o sistema de patentes como um potencializador das doenças negligencias, uma análise diante da manutenção do poder e o acúmulo de capital.

\section{Os instrumentos contextuais de saúde global: a imbricação das doenças negligencias face ao (des)cumprimento da garantia de acesso à medicamentos}

Entende-se atualmente que a saúde se mostra como um desdobramento do direito humano à vida, e desde os tempos mais remotos, se mostra como um direito a ser almejado por todos os indivíduos. Na conjuntura social atual, observa-se que a concretização da saúde está estreitamente relacionado os anseios de todas os momentos históricos que já se vivenciou. De tal modo que, todas as discussões, investimentos e empreendimentos concatenaram para se chegar a denominadores comuns, ou seja, ter saúde no século XXI, envolve acesso a tratamentos médicos e medicamentosos, mas também está relacionado ao meio ambiente físico, como também pelas interações químicas apresentadas. ${ }^{4}$ Considerando a importância dos entornos que envolvem os indivíduos, Schwartz dispõe que:

as pessoas em bom estado de saúde não são as que recebem bons cuidados médicos, mas sim aquelas que moram em casas salubres, comem uma comida sadia, em um meio que lhes permite dar à luz, crescer, trabalhar e morrer. ${ }^{5}$

No entanto, isso nem sempre se faz possível, haja vista toda a configuração de sociedade capitalista na qual se vive em grande parte do globo. Diante disso, se faz importante ter acessos a medicamentos, posto que se a doença não pode ser evitada, a mesma deve ser tratada e, se possível, curada.

Atualmente, não há como tratar de questões de saúde de forma isolada. Problemas locais não encontram mais fronteiras, podendo atingir toda uma coletividade. Isso ocorre principalmente, devido ao fenômeno da globalização. A partir deste fenómeno, novos atores surgiram, remontando um novo cenário no qual merece atenção global, já que a nova configuração de tempo e espaço requer novos empreendimentos. ${ }^{6}$ Tal entendimento, pode ser evidenciado a partir do século XX e início do século XXI, em tal momento passa-se a utilizar-se da terminologia saúde global para questões sanitárias que atingem pessoas além fronteiras, isso calçado no aumento de circulação de pessoas, bens e serviços. Portanto, ações conjuntas devem ser priorizadas para que não se tenha o surgimento de epidemias ou pandemias. ${ }^{7}$

\footnotetext{
4 FORATTINI, Oswaldo Paulo (2000): A saúde pública no século XX. In: Revista de Saúde Pública, São Paulo, v. 34, n. 3, jun. 2000. Tomado de: http://www. scielo.br/scielo.php?script=sci_arttext\&pid=S0034-89102000000300001. Fecha de consulta consulta: 16.06. 2018.

5 SCHWARTZ, Germano André Doederlein (2001). Direito à saúde: efetivação em uma perspectiva sistêmica. Porto Alegre: Livraria do Advogado, pp.40. 6 BROWN, Theodore; CUETO, Marcos; FEE, Elizabeth (2006). A transição de saúde pública 'internacional' para 'global' e a Organização Mundial da Saúde. In: Hist. cienc. saúde-Manguinhos, Rio de Janeiro, v. 13, n. 3, pp. 623-647, Tomado de: http://www.scielo.br/scielo.php?script=sci_arttext\&pid=S010459702006000300005\&lng=en\&nrm=iso, Fecha de consulta: 19.03. 2018.

7 RIBEIRO. Helena (2016): Saúde Global: Olhares do presente, Rio de Janeiro: Ed. Fiocruz. Endereço Eletrônico: nathaliafacco@hotmail.com. ORCID iD:
} 
Sendo assim, ao tratar de saúde global observa-se a existência multidemandas que devem ser atendidas. Não se pode esquecer, que atrelada a elas existem processos políticos, sociais, econômicos e culturais, que interferem diretamente na manutenção da saúde, bem como aparecimento e proliferação de doenças e acesso a medicamentos. ${ }^{8}$ Neste panorama:

Uma questão de saúde global é, em primeiro lugar, aquela que está relacionada com dinâmicas, estruturas e relações políticas no plano internacional. Essas dinâmicas e estruturas internacionais podem ter impacto não só na exposição e vulnerabilidade à doença (ou seja, sobre incidência, prevalência, grupos sociais particularmente afetados), mas também na capacidade de reação e resposta (recursos disponíveis e capacidade de mobilizá-los de forma eficaz). ${ }^{9}$

Frente a toda essa nova estrutura observa-se como ponto central primordial a ação conjunta de vários Estados -Nação, as práticas sanitárias não podem ser tratadas isoladamente. a cooperação internacional não pode ser visualizada como um fardo, posto que as ameaças se mostram comuns, por isso é dever de todos, tais ações não devendo ser causadores de tensões internacionais. ${ }^{10}$ Assim:

A segurança sanitária coletiva é a soma da segurança sanitária individual. A cobertura universal e equitativa de cuidados à saúde é indispensável [...] a segurança individual se soma à segurança coletiva, e a segurança coletiva significa maior segurança individual. ${ }^{11}$

No entanto isso nem sempre se faz possível, principalmente se visualizado pelos direitos de propriedade intelectual, sob o olhar do sistema de patentes. O modelo atual normativo de patentes ilustra fielmente a falta de interesse na colaboração conjunta para a produção de medicamentos, uma vez que elucida privilégios aos inventores que usufruem da exclusividade sobre a fabricação de seus inventos pelo período mínimo de vinte anos, bem como parâmetros rígidos que devem ser seguidos, assegurados pelo principal mecanismo de proteção dos direitos de propriedade intelectual, ou seja, o acordo TRIPs (Acordo sobre Aspectos dos Direitos de Propriedade Intelectual Relacionados ao Comércio). ${ }^{12}$

O TRIPS é um importante acordo internacional, considerado um "acordo-contrato" que se originou principalmente em decorrência do interesse das grandes indústrias farmacêuticas que detinham o monopólio mundial de medicamentos, durante a negociações realizadas na Rodada do Uruguai. O referido acordo, incorporou entre outras disposições, as providencias que já se encontravam na postuladas na convenção

\footnotetext{
https://orcid.org/0000-0003-4465-5802.

8 NUNES, João; VENTURA, Deisy. Apresentação (2016): . In: Lua Nova, São Paulo, n. 98, pp. 7-16, maio./ago. 2016. Tomado de: http://www.scielo.br/scielo. php?script=sci_arttext\&pid=S0102-64452016000200007\&lng=en\&nrm=iso, Fecha de consulta: 11.09 .2018$.

9 NUNES, João; VENTURA, Deisy. Apresentação (2016): op. cit.., pp.7-16.

10 COOPER, Andrew; KIRTON, John; STEVENSON, Michael (2009): Critical cases in global health innovation. In: COOPER, Andrew; KIRTON, John (Org.). Innovation in global health governance: critical cases. Farnham: Ashgate.

11 RIBEIRO. Helena (2016): op.cit., pp.67.

12 WIPO (2018): A Brief History. Tomado de: http://www.wipo.int/about-wipo/en/history.html, Fecha de consulta: 15.02. 2018.
} 
de Paris, bem como na convenção de Berna. ${ }^{13}$ Assim, foi motivo de grandes críticas no cenário internacional, por ter uma estrutura rígida, que ao ser descumprida pode acarretar sanções, favorecendo aqueles que possuem grande capital e tecnologia e, que por consequência, almejam continuar no topo do poder e da lucratividade. Sendo assim, propicia um grande desequilíbrio entre os países do Norte e do Sul social, haja vista a diferença que ambos possuem em pesquisas e desenvolvimento, ao passo que corrobora para que não se consiga um desenvolvimento equânime mundial de medicamentos. ${ }^{14}$

O TRIPs proporcionou a possibilidade que os países membros, ou seja, aquelas que assinaram o acordo, tenham legislações internas acerca da matéria que regulamenta os direitos de propriedade intelectual. No entanto, observa-se claramente, uma espécie de submissão ao que é tratado no acordo internacional, posto que tais legislações não podem contrariar as prerrogativas internacionais já impostas. Neste sentido, ressaltase:

\begin{abstract}
O Acordo TRIPS é um dos três acordos multilaterais que estruturam o Acordo Constitutivo da OMC. A Assinatura dos países nestes Acordos é obrigatória para este ser considerado um Estado-membro. O TRIPS é um tratado-contrato implicando ao país signatário um comprometimento em promulgar leis que internalizem os padrões mínimos constantes no Acordo. Os Estados devem ter em suas legislações nacionais no mínimo o que o acordo da OMC obriga, mas nada impede que possam prever também formas mais restritivas de direitos de propriedade industrial, ou mesmo novas modalidades de direitos de propriedade, tais como os direitos de propriedade intelectual sui generis, sobre plantas, recursos genéticos ou conhecimentos tradicionais a eles associados. ${ }^{15}$
\end{abstract}

Pode-se de tal maneira, afirmar que a estrutura apresentada no acordo TRIPs é influenciada por interesses particulares das grandes multinacionais, que não estão interessadas na cura, nem no tratamento das doenças que assolam grande parte da população mundial. Tais empresas, possuem interesse na fabricação de medicamentos que possibilitam grande potencialidade de lucro, concatenando para que várias populações tenham suas doenças negligenciadas pela falta de poderio econômico que apresentam, bem como, a pouca de lucratividade verificada sobre a venda de tais medicamentos. ${ }^{16}$

A discussão entre direitos de propriedade intelectual, mais especificamente, atrelado

\footnotetext{
13 BARRETO, Ana Cristina Costa (2010): O Direito à saúde e patentes farmacêuticas: o acesso a medicamentos como preocupação global para o desenvolvimento. São Paulo: Revista Aurora. Tomado de: < http://www2.marilia.unesp.br/revistas/index.php/aurora/article/view/1240>, Fecha de consulta: 20.06. 2019.

14 RÊGO, Elba Cristina Lima (2001): Acordo sobre propriedade intelectual da OMC: implicações para a saúde pública nos países em desenvolvimento. In: Revista do BNDES, Rio de Janeiro, v. 8, n. 16, pp. 43-78. Tomado de: https://www.bndes.gov.br/SiteBNDES/export/sites/default/bndes_pt/Galerias/ Arquivos/conhecimento/revista/rev1602.pdf, Fecha de consulta: 18.10.2018.

15 VARELLA, Marcelo Dias; MARINHO, Maria. E. P. A (2005): propriedade intelectual na OMC. Revista do Programa de Mestrado em Direito do UniCEUB, Brasília, v. 2, n. 2, pp.136-153. Tomado de: http://www.egov.ufsc.br/portal/sites/default/files/anexos/22168-22169-1-PB.pdf. Fecha de consulta: 05.07. 2018. pp.142.

16 CHAVES, Gabriela Costa et al (2007): A evolução do sistema internacional de propriedade intelectual: proteção patentária para o setor farmacêutico e acesso a medicamentos. In: Cadernos de Saúde Pública, Rio de Janeiro, v. 2, n. 23, pp. 257-267. Tomado de: http://www.scielo.br/scielo.php?script=sci arttext\&pid=S0102-311X2007000200002. Fecha de consulta: 17.02. 2018.
} 
às patentes de fármacos e o direito a ter acesso à saúde são frequentes e pertinentes de acordo com o cenário social atual, haja vista que, na maioria das vezes, são interesses que se contrapõem. A priori observa-se que as prerrogativas do TRIPs não são favoráveis a população que almeja o tratamento de suas mazelas, já que se mostram segregadas pelos ditames da economia de mercado. Deste modo:

Nessa perspectiva é possível afirmar que existe um forte movimento em curso para tornar o sistema de proteção da propriedade intelectual cada vez mais favorável ao titular da patente, e, por isso, menos sensível ao direito das populações de ter acesso a novas tecnologias. ${ }^{17}$

Devido a essa segregação econômica, as doenças negligenciadas, principalmente aquelas que se encontram nos países em desenvolvimento, não se mostram interessantes as grandes indústrias farmacêuticas multinacionais. Ao passo que, o atual sistema normativo de patentes se mostra como um grade efetivado das desigualdades de acesso a fármacos em todo o mundo, impondo aqueles que não possuem potencial de contraprestação financeira aos fármacos que necessitam, a opção por outros menos eficazes, com efeitos colaterais indesejados, e, em últimos casos, contribui para a impossibilidade de tratamento. ${ }^{18}$

Assuntos atrelados à saúde, acesso a tratamentos e medicamentos são considerados assuntos de saúde pública e deveriam ser tratados com primazia. No entanto, o sistema de patentes impossibilita a inserção de indústrias concorrentes no mercado, acarretando em medicamentos de altos custos e vincula a vida, à saúde o bemestar dos indivíduos ao seu poder de compra. ${ }^{19}$ Pois, seus regramentos interferem diretamente na efetivação do direito à saúde, assim como, em tudo o que a ele se relaciona. Flexibilização das rígidas regras impostas no TRIPs trariam benefícios a mais pessoas. O enfoque deve ser redirecionado, interesses coletivos devem ser postos em primeiro plano e as doenças encontradas nos países em desenvolvimento não seriam deixadas a margem das pesquisas e desenvolvimento. ${ }^{20}$

Conforme aponta o sítio dos médicos sem fronteiras, as doenças negligenciadas se apresentam frente a realidade capitalista vivenciada, ou seja, fazem parte de um sistema estrutural consolidado, consoante ao desinteresse econômico dos grandes fabricantes de medicamentos, já que são:

doenças tratáveis e curáveis que afetam, principalmente, populações com poucos recursos financeiros que, justamente por isso, não despertam o interesse da indústria farmacêutica. Os métodos de tratamento e diagnóstico dessas doenças são antigos e inadequados e demandam investimento em pesquisa e desenvolvimento para se tornarem mais simples e efetivos ${ }^{21}$.

17 CHAVES, Gabriela Costa et al (2007): op. cit., s/p.

18 MEINERS, Constance Marie Milward de Azevedo (2008). Patentes farmacêuticas e saúde pública: desafios à política brasileira de acesso ao tratamento anti-retroviral. In: Cad. Saúde Pública, Rio de Janeiro, v. 24, n. 7, pp.1467-1478. Tomado de: http://www.scielo.br/scielo.php?pid=S0102311X2008000700002\&script=sci_abstract\&tlng=pt, Fecha de consulta: 07.02.2019.

19 MEINERS, Constance Marie Milward de Azevedo (2008): op.cit., pp.1467-1478.

20 CORREA, Carlos (2005): O Acordo TRIPS e o acesso a medicamentos nos países em desenvolvimento. In: Sur, Rev. int. direitos human, São Paulo, v. 2 , n. 3. Tomado de: http://ref.scielo.org/f838wh. Fecha de consulta: 01.02. 2018.

21 MÉDICOS SEM FRONTEIRAS (2012): O assunto é doenças negligenciadas. Tomado de: https://www.msf.org.br/noticias/o-assunto-e-doencas- 

LIMITES E POSSIBILIDADES NA PERSPECTVA DA SAÚDE GLOBAL

Como pode-se observar, as doenças negligenciadas fazem parte do século XXI, merecem atenção e devem sim possuir tratamentos adequados. Ao passo, como já explanado, as doenças não podem mais serem tratadas de forma isoladas, pois suas consequências, ainda que sentidas de formas distintas serão globais. Portanto, o segundo capítulo ilustrará o entrelaçamento do sistema de patentes e das doenças negligencias, sob um olhar crítico a respeito do desequilíbrio tecnológico e inovador, bem como os principais motivos que corroboram para que esta estrutura ainda se mantenha.

\section{O sistema de patentes como um potencializador das doenças negligencias: uma análise diante da manutenção do poder e do acúmulo de capital}

A realidade contemporânea da saúde global encontra-se revestida por estatísticas espantosas, as quais trazem à baila a necessidade de medidas urgentes capazes de garantir o efetivo acesso à saúde, bem como, a igualdade na distribuição de medicamentos. Dentre essas estatísticas a Organização Mundial da Saúde (OMS) dispõe sobre as doenças negligenciadas, que afetam mais de 1 bilhão de pessoas mundialmente, primordialmente pessoas em situação de extrema pobreza fator que potencializa o desinteresse da indústria farmacêutica em pesquisar e produzir os medicamentos. ${ }^{22}$

Sendo assim, a Organização Mundial da Saúde (OMS) intitulou algumas doenças como negligenciadas, as quais sejam: dengue, raiva, hanseníase, doença de chagas, leishmaniose, entre outras. As supracitadas doenças tornaram-se negligenciadas devido ao descaso com a pesquisa e a inadequada distribuição de investimentos para a produção dos respectivos medicamentos, pois conforme demonstrado em pesquisas apenas $1 \%$ dos fármacos submetidos à registros destinam-se a satisfação social e aplicabilidade das doenças negligenciadas. ${ }^{23}$

Além disso, outro potencializador do crescimento exponencial das doenças negligenciadas encontra-se no instituto da propriedade intelectual, mais precisamente, nas patentes de medicamentos, ao passo que o Estado confere monopólio à indústria farmacêutica, ou seja, intitula que a mesma seja titular exclusiva da exploração e, em teoria, nesse lapso temporal recupera o investimento realizado para desenvolver a pesquisa e reproduzir o desenvolvimento. Sob outra vertente, a patente consagra a exceção a livre concorrência e a liberdade de iniciativa, favorecendo mercado oligopsônicos. ${ }^{24}$

Desde a sua criação, os direitos de propriedade intelectual foram entendidos como um sistema de regras e princípios, que regulamentam a aquisição, uso e interesses face a matéria prima intangível e suscetível de comercialização. Frente a isso, a invenção e sua patente considerada como uma espécie de propriedade foram imbricadas na necessidade de que toda e qualquer forma de propriedade atendessem a sua função social. ${ }^{25}$

\footnotetext{
negligenciadas. Fecha de consulta: 07.02. 2019. s/p

22 OMS. Organização Mundial da Saúde (2010): Primeiro relatório da OMS sobre as doenças tropicais negligenciadas, pp.41.

23 CHIRAC, P.; TORREELE, E (2006): Global framework on essential health R\&D. The Lancet, Volume 367, Issue 9522, pp.1560.

24 BARBOSA, D. B (2010): Uma introdução à propriedade intelectual. Lumen Juris, pp.1-951. Tomado de: http://www.denisbarbosa.addr.com/arquivos/ livros/umaintro2.pdf, Fecha de consulta: 20.09. 2019.

25 BULHÕES, E. P (2008): O papel das redes transnacionais de ONGs no contencioso das patentes farmacêuticas entre Brasil e Estados Unidos. $244 f .2008$. Dissertação (Mestrado em Relações Internacionais). Porto Alegre: UFRS, pp. 125
} 
Ocorre que, a salvaguarda do direito à propriedade intelectual interfere na obrigação estatal de garantir a implementação do acesso à medicamentos. ${ }^{26}$ As patentes de fármacos e a garantia ao acesso de medicamentos encontram-se imbricados ao aspecto econômico, possuindo influência direta nas vertentes das políticas públicas, ao passo que necessitam de unificação do desenvolvimento internacional e nacional com a finalidade de propiciar a repartição equânime ao bem estar social e econômico. ${ }^{27}$

O ausente desenvolvimento internacional e nacional e a ineficácia do sistema de patentes perante a pesquisa de medicamentos voltados para as doenças negligenciadas encontra entrave no desinteresse das farmacêuticas, devido ao pequeno percentual lucrativo que incentive a criação de medicamentos mais eficientes. Assim, as doenças negligenciadas nada mais são que doenças "esquecidas", devido a omissão estatal e dos grupos farmacêuticos perante a parcela populacional sem situação financeira condizente para adquirir o necessário medicamento, bem como ao contexto econômico precário de contrapartida financeira. ${ }^{28}$

Confirma-se que, a indústria farmacêutica preocupa-se prioritariamente com o desenvolvimento voltado para áreas estratégicas financeiramente, rechaçando a inovação para as doenças negligenciadas. Na verdade, a inovação e o plano tecnológico se interessam em investir nas áreas declaradas estratégicas governamentalmente, pois a lógica de investimento classifica-se como puramente econômica. Ademais, a vertente econômica propaga a utilização de tratamentos declarados como desumanos e, até mesmo, degradantes, face a utilização de métodos antiquados e o descrédito com a população mais pobre, que são afastados pela indústria e pelo governo de seus direitos de cidadão, situação que faz com que sejam até mesmo intitulados como não cidadãos, diante da lógica perversa da falta de inovação. ${ }^{29}$

Diante disso, podemos trazer à baila a Doença de Chagas, que atinge mais de um quarto da população localizada na América Latina, o medicamento utilizado para seu tratamento é o Benzonidazol. No entanto, a utilização ilimitada do medicamento resulta em doenças potencializadas colateralmente..$^{30}$

Quanto a Malária, os medicamentos mantiveram o padrão de produção imposto na década de 40 , onde estipulou doses específicas para cada fase da doença e ciclo do protozoário. Porém, os medicamentos apenas tinham efeito contra a espécie específica causadora da doença ${ }^{31}$. Com o aprimoramento, os fármacos apresentaram deficiências, ou seja: "resistência ao medicamento Cloroquina, [...] a qual chega a mais de $90 \%$ em algumas partes do mundo.[...] as terapias de combinação podem ser caras e possuir esquemas terapêuticos complicados. Os países que mais sofrem não tem capacidade para disponibilizar os medicamentos". ${ }^{32}$

26 PIOVESAN, F (2007). Direitos humanos e propriedade intelectual. Cultura livre, pp. 20.

27 BARRETO, A. C. C (2011): A Flexibilização do Acordo TRIPS e a Necessidade de Respeito aos Direitos Humanos nas Regras da OMC. $136 f .2011$. Dissertação (Mestrado em Relações Internacionais) - Universidade Estadual da Paraíba, João Pessoa, pp.16.

28 BRASIL (2014): Ministério da Saúde. Agência fiocruz de notícias: doenças negligenciadas. Tomado de: http://www.agencia.fiocruz.br/doen\%C3\%A7asnegligenciadas, Fecha de consulta: 06.09.2019.

29 TRESSE Vitor Schettino (2015): Doenças negligenciadas e patentes de fármacos: uma análise da garantia ao direito à saúde através do novo paradigma colaborativo. (Dissertação de mestrado), Rio de Janeiro, pp.1-112. Tomado de: http://www.bdtd.uerj.br/tde_busca/arquivo.php?codArquivo=9203, Fecha de consulta: 20.09.2019.

30 TRESSE Vitor Schettino (2015): op.cit., pp.42-57.

31 FRANÇA, Tanos C.C.; SANTOS, Marta G. dos; VILLAR, José de Figueiroa (2008):.Malária: Aspectos e Quimioterapia. Química Nova, São Paulo, v.31, n.5, 2008. Tomado de:

http://www.scielo.br/scielo.php?pid=S0100-40422008000500060\&script=sci_arttext, Fecha de consulta: 03.10.2019.

32 DRUGS FOR NEGLECTED DISEASES INITIATIVE (2013): Annual report 2013: a decade of r\&d for neglected patients. Tomado de: 

LIMITES E POSSIBILIDADES NA PERSPECTVA DA SAÚDE GLOBAL

A Leishmaniose liderou como a doença mais comum mundialmente, encontrada entre 700 mil a 1 milhão de infectados anualmente. O fármaco para seu tratamento foi descoberto na década de 40, perpassando o lapso temporal até a atualidade, sendo utilizado, inclusive, com o alto índice de falha e sua toxidade. A doença mesmo não ocasionando morte, mas propaga a desigualdade e a exclusão social, diante das manchas espalhadas pelo corpo do enfermo. ${ }^{33}$

No tocante à Hanseníase, o seu demérito social inclui vertentes econômicas, políticas e sociais. Pois, o enfermo é permeado por preconceito, medo e exclusão, essa situação potencializa-se face ao contexto histórico da enfermidade, diante das concepções criadas equivocadamente pelos membros da sociedade, ao passo que induziu a necessidade do detentor da doenças ser isolado socialmente. A situação permeou no tempo que, inclusive, atualmente os portadores da hanseníase escondem o diagnóstico positivo, submetendo-se inclusive a morte com a finalidade de evitar a rejeição e exclusão comunitária. ${ }^{34}$

Nessa senda, os tratamentos desumanos e, até mesmo, degradantes, inclusive, com efeitos colaterais devastadores para os pacientes, são uma das facetas das doenças negligenciadas. Como percebe-se os instrumentos de propriedade intelectual, mais precisamente, o sistema de patentes está imbricado na propagação das doenças negligenciadas, sob o prisma do sistema econômico-financeiro das indústrias farmacêuticas. O regime atual de patentes fez emergir inúmeras controvérsias, ao passo que colaborou para a eternização de supracitadas doenças, à medida que os avanços tecnológicos deram origem a potentes instrumentos maquinários capazes o efeito de suas incidências mas, em contrapartida, não são utilizadas com essa finalidade devido à problemática envolvendo a contrapartida financeira. ${ }^{35}$

As dificuldades respaldam-se diante dos altos preços dos medicamentos, que são estipulados face a larga escala demandada pelos padrões da classe social alta, ou seja, a população rica, com capacidade financeira para arcar com os custos dos medicamentos. Assim, dependendo do diagnóstico da doença, a classe social baixa, ou seja, a população mais pobres não possui recursos financeiros capazes de financiar as medicações. Sendo assim, as doenças negligenciadas, classificadas muitas vezes como de alto risco e de fácil disseminação, não são consideradas rentáveis para as indústrias farmacêuticas, trazendo à baila a problemática da saúde pública que enquadram as referidas doenças no Hiato dos 10/90, onde $90 \%$ das ciência investigativa das farmacêuticas voltam-se para amparar, apenas, $10 \%$ da população mundial. ${ }^{36}$

Conforme o exposto, as doenças intituladas como negligenciadas enquadram-se na classificação de doenças "esquecidas", devido a inércia estatal e dos grupos farmacêuticos frente a parcela populacional de condição financeira ínfima para adquirir

\footnotetext{
$<$ http://www.dndi.org/images/stories/annual_report/2013/DNDi_AR_2013.pdf>, Fecha de consulta: 28.09.2019.

33 BARBIERI, Carolina Luisa Alves; MARQUES, Heloísa Helena de Sousa (2009): Hanseníase em crianças e adolescentes: revisão bibliográfica e situação atual no Brasil. Pediatria, São Paulo, v.31, n.4, pp. 281-290. Tomado de: http://www.pediatriasaopaulo.usp.br/upload/pdf/1319.pdf, Fecha de acesso: 03.02.2019.

34 BAIALARDI, Katia Salomão(2007): O estigma da hanseníase: relato de uma experiência em grupo com pessoas portadoras. Hansenologia Internationalis, Bauru, v.32, n.1. Tomado de: http://periodicos.ses.sp.bvs.br/scielo.php?script=sci_arttext\&pid=S1982- 51612007000100004\&lng=pt\&nrm=iso=pt, Fecha de consulta: 03.02.2019.

35 POGGE, Thomas (2008): Medicamentos para o mundo: incentivando a inovação sem obstruir o acesso livre. In: Revista SUR, São Paulo, v. 5, n 8. Tomado de: http://www.surjournal.org/conteudos/pdf/8/pogge.pdf, Fecha de consulta: 11.04. 2019.

36 POGGE, Thomas (2008): op. cit. s/p.
} 
o necessário medicamento. Da mesma forma, a perspectiva econômica desacredita a população negligenciada, ao passo que utiliza de métodos antiquados e, até mesmo, medicamentos ineficazes sob o pretexto perverso da falta de inovação.

\section{Conclusão}

Sem intenção de esgotar a temática abordada, o presente artigo teve por intuito compreender melhor o entrelaçamento do atual sistema de patentes, com o acesso a medicamentos e a incidência de doenças negligenciadas após o fenômeno da globalização. A saúde, nos mais distintos contextos históricos se faz motivo de atenção e preocupação para os indivíduos. O que não se faz diferente atualmente, entretanto, a modificação se dá no sentido de que não se pode mais falar, estudar e tratar de questões relacionadas a saúde de forma isolada, posto que, as fronteiras cada vez mais inexistem, transformando problemas locais em globais, necessitando de ações mais amplas e conjuntas para soluções efetivas, principalmente após o fenômeno da globalização.

A saúde, analisada sob a ótica global requer inúmeros investimentos em todos os setores, inclusive em pesquisas, desenvolvimento e tecnologias, onde olhares e interesses egoísticos devem ser deixados de lado. Entretanto, isso não ocorre de forma equânime, haja vista que, parte do tratamento de determinadas doenças, a exemplo das negligenciadas, não despertam interesse das grandes indústrias farmacêuticas. Tal assertiva se baseia na afirmação de que, o lucro e interesses individuais tem se sobreposto a interesses coletivos, ou seja, a saúde de determinados indivíduos despertam maiores investimentos do que as doenças que assolam outros que possuem menor possibilidade de compra. Atenta-se, que tal situação encontra respaldo no atual sistema de patentes e as prerrogativas rígidas conferidas pelo acordo TRIPS.

Observa-se que, o inventor ao dispor de privilégios de exclusividade sobre seus inventos, segrega o alcance de medicamentos para determinados segmentos sociais, bem como desestimula a inserção de novas indústrias e de concorrência no mercado farmacêutico, pois o acordo TRIPs contribui para manutenção do monopólio das grandes empresas multinacionais, com seus padrões rígidos de obediência. As doenças negligenciadas, normalmente se mostram nessa condição, entre outros motivos devido a negligência das grandes indústrias, a exemplo disso, tem-se a doença de Chagas, Malária, leishmaniose, hanseníase, as quais são doenças que atingem em sua maioria a população de países pobres e em desenvolvimento, concatenando para o entendimento de que o poderio econômico dos indivíduos se mostra como potencializador de investimentos.

As doenças esquecidas, são reflexo da conjuntura de mercado que prioriza o lucro à coletividade. Entretanto, visualiza-se que medidas mais eficazes devem ser tomadas, uma vez que, ainda que atinjam populações específicas, terão reflexos globais. O atual sistema de patentes se mostra inconsistente com a realidade do século XXI, exigindo novas respostas para velhas demandas. 


\section{REFERÊNCIAS BIBLIOGRÁFICAS}

- BAIALARDI, Katia Salomão(2007): O estigma da hanseníase: relato de uma experiência em grupo com pessoas portadoras. Hansenologia Internationalis, Bauru, v.32, n.1. Tomado de: http://periodicos.ses.sp.bvs.br/scielo.php?script=sci_arttext\&pid=S198251612007000100004\&lng=pt\&nrm=iso=pt, Fecha de consulta: 03.02.2019.

- BARBIERI, Carolina Luisa Alves; MARQUES, Heloísa Helena de Sousa (2009): Hanseníase em crianças e adolescentes: revisão bibliográfica e situação atual no Brasil. Pediatria, São Paulo, v.31, n.4, pp. 281-290. Tomado de: http://www.pediatriasaopaulo.usp.br/upload/ pdf/1319.pdf, Fecha de acesso: 03.02.2019.

- BARBOSA, D. B (2010): Uma introdução à propriedade intelectual. Lumen Juris, pp.1951. Tomado de: http://www.denisbarbosa.addr.com/arquivos/livros/umaintro2.pdf, Fecha de consulta: 20.09. 2019.

- BARRETO, A. C. C (2011): A Flexibilização do Acordo TRIPS e a Necessidade de Respeito aos Direitos Humanos nas Regras da OMC. 136f. 2011. Dissertação (Mestrado em Relações Internacionais) - Universidade Estadual da Paraíba, João Pessoa, pp.16.

- BARRETO, Ana Cristina Costa (2010): O Direito à saúde e patentes farmacêuticas: o acesso a medicamentos como preocupação global para o desenvolvimento. São Paulo: Revista Aurora. Tomado de: < http://www2.marilia.unesp.br/revistas/index.php/aurora/ article/view/1240>, Fecha de consulta: 20.06. 2019.

- BRASIL (2014): Ministério da Saúde. Agência fiocruz de notícias: doenças negligenciadas. Tomado de: http://www.agencia.fiocruz.br/doen\%C3\%A7as-negligenciadas, Fecha de consulta: 06.09.2019.

- BROWN, Theodore; CUETO, Marcos; FEE, Elizabeth (2006). A transição de saúde pública 'internacional' para 'global' e a Organização Mundial da Saúde. In: Hist. cienc. saúdeManguinhos, Rio de Janeiro, v. 13, n. 3, pp. 623-647, Tomado de: http://www.scielo.br/ scielo.php?script=sci_arttext\&pid=S0104-59702006000300005\&lng=en\&nrm=iso, Fecha de consulta: 19.03. 2018.

- BULHÕES, E. P (2008): O papel das redes transnacionais de ONGs no contencioso das patentes farmacêuticas entre Brasil e Estados Unidos. 244f. 2008. Dissertação (Mestrado em Relações Internacionais). Porto Alegre: UFRS, pp. 125.

- CHAVES, Gabriela Costa et al (2007): A evolução do sistema internacional de propriedade intelectual: proteção patentária para o setor farmacêutico e acesso a medicamentos. In: Cadernos de Saúde Pública, Rio de Janeiro, v. 2, n. 23, pp. 257-267. Tomado de: http:// www.scielo.br/scielo.php?script=sci_arttext\&pid=S0102-311X2007000200002. Fecha de consulta: 17.02. 2018.

- CHIRAC, P.; TORREELE, E (2006): Global framework on essential health R\&D. The Lancet, Volume 367, Issue 9522, pp.1560.

- COOPER, Andrew; KIRTON, John; STEVENSON, Michael (2009): Critical cases in global health innovation. In: COOPER, Andrew; KIRTON, John (Org.). Innovation in global health governance: critical cases. Farnham: Ashgate.

- CORREA, Carlos (2005): O Acordo TRIPS e o acesso a medicamentos nos países em desenvolvimento. In: Sur, Rev. int. direitos human, São Paulo, v. 2, n. 3. Tomado de: http:// ref.scielo.org/f838wh. Fecha de consulta: 01.02. 2018.

- DRUGS FOR NEGLECTED DISEASES INITIATIVE (2013): Annual report 2013: a decade of r\&d for neglected patients. Tomado de: <http://www.dndi.org/images/stories/annual_report/2013/DNDi_AR_2013.pdf>, Fecha de consulta: 28.09.2019.

- FORATTINI, Oswaldo Paulo (2000): A saúde pública no século XX. In: Revista de Saúde Pública, São Paulo, v. 34, n. 3, jun. 2000. Tomado de: http://www.scielo.br/scielo. php?script=sci_arttext\&pid=S0034-89102000000300001. Fecha de consulta consulta: 
16.06. 2018.

- FRANÇA, Tanos C.C.; SANTOS, Marta G. dos; VILLAR, José de Figueiroa (2008):.Malária: Aspectos e Quimioterapia. Química Nova, São Paulo, v.31, n.5, 2008. Tomado de: http:// www.scielo.br/scielo.php?pid=S0100-40422008000500060\&script=sci_arttext, Fecha de consulta: 03.10.2019.

- MÉDICOS SEM FRONTEIRAS (2012): O assunto é doenças negligenciadas. Tomado de: https://www.msf.org.br/noticias/o-assunto-e-doencas-negligenciadas. Fecha de consulta: 07.02. 2019. s/p.

- MEINERS, Constance Marie Milward de Azevedo (2008). Patentes farmacêuticas e saúde pública: desafios à política brasileira de acesso ao tratamento anti-retroviral. In: Cad. Saúde Pública, Rio de Janeiro, v. 24, n. 7, pp.1467-1478. Tomado de: http://www.scielo.br/ scielo.php?pid=S0102-311X2008000700002\&script=sci_abstract\&tlng=pt, Fecha de consulta: 07.02.2019.

- NUNES, João; VENTURA, Deisy. Apresentação (2016): . In: Lua Nova, São Paulo, n. 98, pp. 7-16, maio./ago. 2016. Tomado de: http://www.scielo.br/scielo.php?script=sci_ arttext\&pid=S0102-64452016000200007\&lng=en\&nrm=iso, Fecha de consulta: 11.09.2018.

- OMS. Organização Mundial da Saúde (2010): Primeiro relatório da OMS sobre as doenças tropicais negligenciadas, pp.41.

- PIOVESAN, F (2007). Direitos humanos e propriedade intelectual. Cultura livre, pp. 20.

- POGGE, Thomas (2008): Medicamentos para o mundo: incentivando a inovação sem obstruir o acesso livre. In: Revista SUR, São Paulo, v. 5, n 8. Tomado de: http://www.surjournal.org/conteudos/pdf/8/pogge.pdf, Fecha de consulta: 11.04. 2019.

- RÊGO, Elba Cristina Lima (2001): Acordo sobre propriedade intelectual da OMC: implicações para a saúde pública nos países em desenvolvimento. In: Revista do BNDES, Rio de Janeiro, v. 8, n. 16, pp. 43-78. Tomado de: https://www.bndes.gov.br/SiteBNDES/ export/sites/default/bndes_pt/Galerias/Arquivos/conhecimento/revista/rev1602.pdf, Fecha de consulta: 18.10.2018.

- RIBEIRO. Helena (2016): Saúde Global: Olhares do presente, Rio de Janeiro: Ed. Fiocruz.

- SCHWARTZ, Germano André Doederlein (2001). Direito à saúde: efetivação em uma perspectiva sistêmica. Porto Alegre: Livraria do Advogado, pp.40.

- TRESSE Vitor Schettino (2015): Doenças negligenciadas e patentes de fármacos: uma análise da garantia ao direito à saúde através do novo paradigma colaborativo. (Dissertação de mestrado), Rio de Janeiro, pp.1-112. Tomado de: http://www.bdtd.uerj.br/ tde_busca/arquivo.php?codArquivo=9203, Fecha de consulta: 20.09.2019.

- VARELLA, Marcelo Dias; MARINHO, Maria. E. P. A (2005): propriedade intelectual na OMC. Revista do Programa de Mestrado em Direito do UniCEUB, Brasília, v. 2, n. 2, pp.136-153. Tomado de: http://www.egov.ufsc.br/portal/sites/default/files/anexos/22168-22169-1PB.pdf. Fecha de consulta: 05.07. 2018. pp.142.

- WIPO (2018): A Brief History. Tomado de: http://www.wipo.int/about-wipo/en/history. html, Fecha de consulta: 15.02. 2018. 